\title{
Direct searches for a cosmological stochastic gravitational wave background
}

\author{
Albert Lazzarini ${ }^{\dagger}$ \\ LIGO Laboratory, California Institute of Technology, Pasadena, CA 91125 USA \\ email: lazzarin@caltech.edu \\ †n behalf of the LIGO Scientific Collaboration
}

\begin{abstract}
LIGO has completed a series of observations searching for evidence of a stochastic gravitational wave background. There has been no detection to date. However the sensitivity of the observations is approaching the Big Bang nucleosynthesis bound. Future observations will provide information that bounds a number of early Universe models of stochastic gravitational waves.
\end{abstract}

Keywords: Stochastic gravitational wave background, SGWB, cosmological gravitational waves PACS: $95.30 . \mathrm{Sf}, 95.55 . \mathrm{Ym}, 95.85 . \mathrm{Sz}, 98.80 .-\mathrm{k}$, 98.80.Qc

\section{INTRODUCTION}

A stochastic background of gravitational waves results from the random superposition of an extremely large number of unresolved and independent gravitationalwave (GW) emission events. Such a background is analogous to the cosmic microwave background radiation (CMBR), though its spectrum is unlikely to be thermal. The emission process could be the result of cosmological processes, as with the CMBR, but occurring much earlier after the big bang-e.g., during inflation. Placing upper limits, and eventually detecting, the energy density of a stochastic background of gravitational waves is one of the long term goals of GW detectors.

In addition to cosmological sources of stochastic GWs, the incoherent superposition of many signals from a variety of astrophysical objects can also lead to foreground stochastic GWs. These signal sources include coalescing binaries, supernovae, pulsars, low mass Xray binaries (LMXBs), newly born neutron stars (normal modes - $\mathrm{R}$ modes), binary black holes. The spectra of GWs produced in this manner follow from characteristics of individual sources [1][2][3]. It is also possible that the GW background does not have a Gaussian character. Depending on source rates, the stochastic component could be more characterized by a non-Gaussian (popcorn noise) nature [4]. However, this paper will focus on a cosmological stochastic GW background.

The stochastic background spectrum is typically characterized in terms of a dimensionless quantity $\Omega_{\mathrm{gw}}(f)-$ the GW energy density per unit logarithmic frequency, normalized by the critical energy density $\rho_{c}$ needed to close the universe. Previous direct measurements of a stochastic background, in the $\sim 10 \mathrm{~Hz}$ to $\sim 10^{4} \mathrm{~Hz}$ frequency band accessible to earth-based detectors, have been limited to establishing upper limits on $\Omega_{\mathrm{gw}}(f) \gg 1$, with a published result from LIGO's first science run (S1) of $\Omega_{\mathrm{gw}}<46$ [5]. Recent results submitted for publication [6] by the LIGO Scientific Collaboration (LSC) from the $\mathrm{S} 3$ science run report much tighter upper limits on a stochastic GW background in the frequency band $60-250 \mathrm{~Hz}$, namely $\Omega_{\mathrm{gw}}<8.4 \times 10^{-4}$. In terms of GW energy density, the S3 limits are approximately five orders of magnitude below our previous S1 limit in the terrestrial frequency band.

LIGO is composed of three interferometers at two sites: $4 \mathrm{~km} \mathrm{H1}$ and $2 \mathrm{~km} \mathrm{H2}$ detectors, are collocated at Hanford, WA; and a $4 \mathrm{~km} \mathbf{L 1}$ detector, located in Livingston, LA. Each detector is a power-recycled Michelson interferometer, with long Fabry-Perot cavities in each of its orthogonal arms. The LIGO detection band spans the decades $40 \mathrm{~Hz} \lesssim f \lesssim 4 \mathrm{kHz}$. These interferometers are sensitive to quadrupolar oscillations in the space-time metric due to a passing $\mathrm{GW}$, and therefore capable of measuring directly the GW strain amplitude, $h(t)$. The detector configuration and performance during LIGO's first science run (S1) is described in [7].

Fig. 1 shows reference amplitude spectra of equivalent strain noise for the S3 run.

\section{THE STOCHASTIC GRAVITATIONAL WAVE BACKGROUND}

\section{Characterization}

Fig. 2 shows a schematic representation of the epoch of inflation during which relic GWs detectable today would have been produced. Such relic waves constitute a 


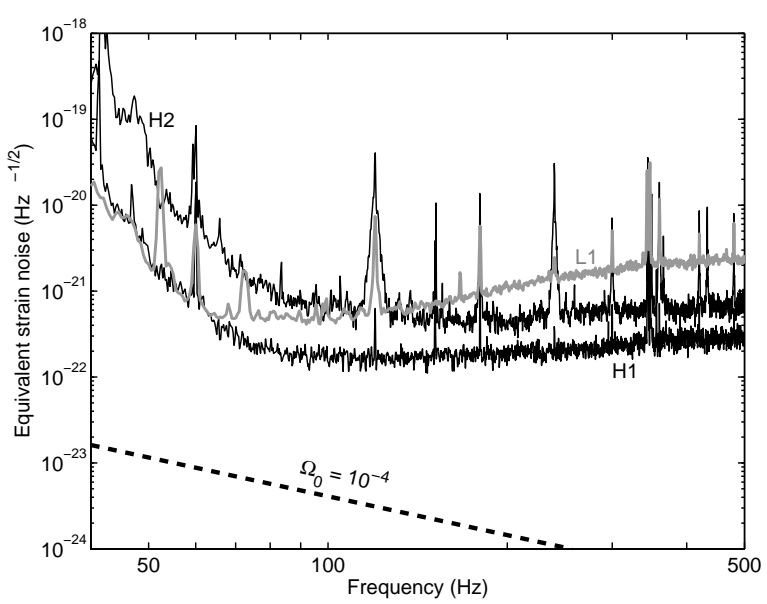

FIGURE 1. Reference sensitivity curves during the S3 data run, in terms of equivalent strain noise density. Also shown is the $f^{-1.5}$ strain noise level corresponding to a constant stochastic background, $\Omega_{0}=10^{-4}$.

weak stochastic GW background. The energy density in $\mathrm{GW}$ is related to the time derivative of measurable strain via the relation:

$$
\rho_{g w}=\frac{c^{2}}{32 \pi G} \overline{\dot{h}_{i j}(t) \dot{h}_{i j}(t)} .
$$

In the literature [8][9][10], the GW spectrum has been characterized in terms of logarithmic frequency derivative of $\rho_{g w}$,

$$
\Omega_{g w}(f) \equiv \frac{1}{\rho_{c}} \frac{d \rho_{g w}(f)}{d \ln f},
$$

where $\rho_{c}$ is the critical closure density of the Universe. $\Omega_{g w}(f)$ is directly related to the strain spectral density measurable by an interferometer,

$$
S_{g w}(f)=\frac{4 G \rho_{c}}{5 \pi c^{2}} \frac{\Omega_{g w}(f)}{f^{3}} .
$$

The strain scale is given by

$\widetilde{h}(f)=\sqrt{S_{h}(f)}=4 \times 10^{-22} \sqrt{\Omega_{g w}(f)}\left(\frac{100 \mathrm{~Hz}}{f}\right)^{3 / 2} \mathrm{~Hz}^{-1 / 2}$.

\section{Possible cosmological sources}

There are a number of possible cosmological sources of GWs:

- Inflation

- First order phase transitions

- Cosmic strings

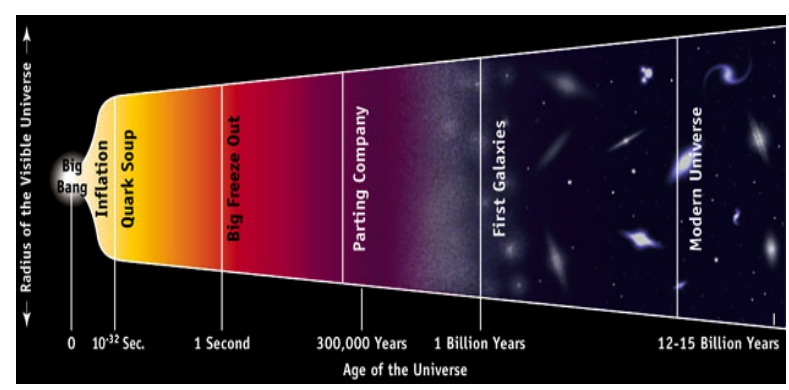

Source: Adapted from Caldwell \& Kamionkowski[11].

FIGURE 2. Cosmological GWs are expected to be produced during the inflationary period of the Universe's history

\section{- Pre-Big-Bang physics}

Fig. 3 presents a landscape of the stochastic GW background spanning many orders of magnitude in both frequency and strength. The various mechanisms and sources noted in the figure are discussed below.

The inflationary model [12] predicts that (i) the Universe is spatially flat, (ii) implying a very nearly scaleinvariant spectrum of Gaussian density perturbations, and (iii) a nearly flat spectrum of gravitational waves: $\Omega_{g w}(f) \sim$ const [13]. The maximum value of $\Omega_{g w}$ within this model is determined by the quadrupole moment of the CMB. The COBE data imply that the spectrum of GWs at the lowest frequencies is given by[14]:

$$
\begin{aligned}
& \Omega_{g w}(f) \leq 1.4 \times 10^{-10}\left(H_{0} / f\right)^{2} ; H_{0} \lesssim f \lesssim 30 H_{0} \\
& \Omega_{g w}(f) \lesssim 2 \times 10^{-13} ; f \gtrsim 30 H_{0} .
\end{aligned}
$$

The Hubble constant is given by $H_{0} \equiv 100 \times$ $h_{100} \mathrm{~km} \mathrm{~s}^{-1} \mathrm{Mpc}^{-1} \approx 71 \mathrm{~km} \mathrm{~s}^{-1} \mathrm{Mpc}^{-1}$. Fig. 3 shows this inflationary limit.

GWs detectable today in the LIGO band were generated at an earlier epoch at much shorter wavelengths. This can be understood by considering Fig. 4. This is a log-log plot of time vs. wavelength or scale. As the Universe expands, the horizon (Hubble length) grows linearly with time. On the other hand, the cosmological expansion causes radiation produced at an earlier epoch to be red-shifted over time. During the matter-dominated phase, this red-shift scales as $t^{2 / 3}$; at earlier epochs it scales as $t^{1 / 2}$. The radiation dominated-matter dominated transition occurs at

$$
z_{e q} \sim 7000 \rightarrow t_{e q} \sim 10^{12} \mathrm{~s} .
$$

GWs produced during inflation correspond to the epoch $t \sim 10^{-23}-10^{-24} \mathrm{~s}$, when $\lambda_{g w} \sim c / H \sim 10^{-15} \mathrm{~m}$.

First order phase transitions in the early Universe produced by colliding bubbles or hydrodynamic turbulence 


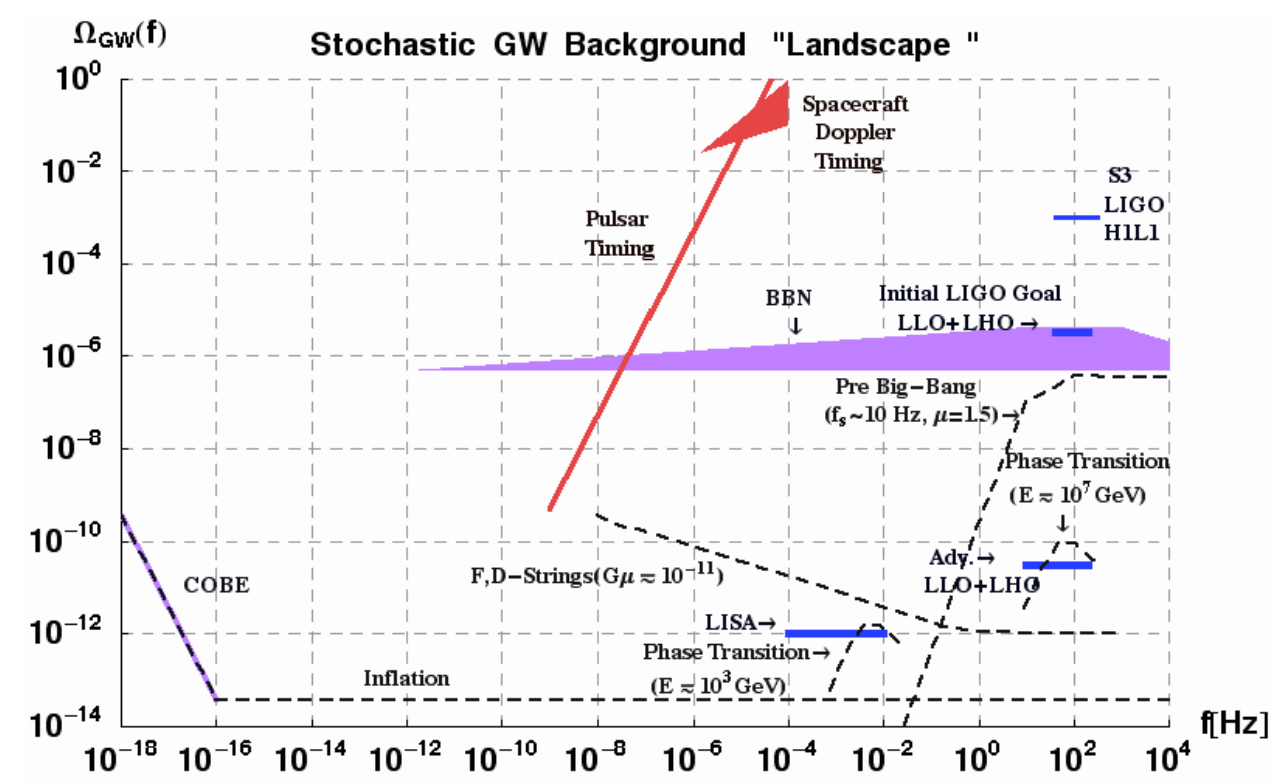

FIGURE 3. Log-log map of the stochastic GW background: sources and measurements at different $f_{g w}$. Energy scales for first order phase transitions that correspond to the LIGO and LISA bands are $10^{7}$ and $10^{3} \mathrm{GeV}$, respectively. Cosmological strings would produce the indicated spectrum for string tension $G \mu \sim 10^{-11}$. Included in the plot are a number of projected interferometric sensitivities (H1-L1 at design $\sim 10^{-6}$; LISA $\sim 10^{-12}$; Advanced LIGO $\sim 10^{-11}$ ) and recently measured H1-L1 result for the S3 science run $\left(\Omega_{g w} \leq 8.4 \times 10^{-4}\right.$ at $90 \%$ C.L. $)$.

having a characteristic energy scale $E^{*}$ are capable of producing GWs [15][16]. The other parameters of the theory are the expansion velocity of the bubble walls, $\beta$, and the Hubble parameter at the time of the phase transition, $H_{*}$. GWs produced by this mechanism would exhbit a frequency-dependent structure peaking at characteristic frequency

$$
f_{g w}^{\text {today }} \sim 10^{-8} \frac{\beta}{H_{*}} \frac{E^{*}}{1 G e V}
$$

The LIGO detection band and $\frac{\beta}{H_{*}} \sim 10^{3}$ corresponds to transition energies $E^{*} \sim 10^{7} \mathrm{GeV}$ and to peak values $\Omega_{g w}\left(f_{L I G O}\right) \sim 10^{-10}$. Refer to Fig. 3 .

Cosmological strings are also putative sources of copious GWs [17]. The main parameters of the theory are the string tension, $G \mu \sim 10^{-11}$ and the conversion efficiency to $\mathrm{GWs}, \varepsilon \sim 10^{-1}$. These lead to a spectrum of GWs,

$$
\Omega_{g w}(f) \propto(G \mu)^{-2 / 3}\left(f H_{0}\right)^{-1 / 3},
$$

where $H_{0}$ is the Hubble constant. Refer to Fig. 3.

Last, a class of scenarios termed pre-big-bang models [18][19][20] consider the dynamic evolution of perturbations that are amplified by accelerated evolution from a cold flat initial regime to a final hot highly curved regime that is marked as the beginning of the post-big-bang epoch. In [20], this model produces a spectrum of GWs that increases with increasing frequency. The model is parametrized by the frequency scale, $f_{s}$, determining the increasing portion of the spectrum. Above a cutoff frequency, $f_{1}$, the spectrum decreases rapidly. This model gives rise to

$$
\begin{aligned}
& \Omega_{g w}(f) \propto f^{3} \text { for } f<f_{s} \text { and } \\
& \Omega_{g w}(f) \quad \sim \text { const for } f_{1}>f>f_{s} \\
& \Omega_{g w}(f) \quad \rightarrow 0 \text { for } f>f_{1} .
\end{aligned}
$$

\section{Experimental limits on the strength of the stochastic GW background}

As was discussed above, at the lowest frequencies, the COBE data limit $\Omega_{g w}(f) \lesssim 10^{-13}$ at frequencies $f \sim H_{0}$ [14]. Next, pulsar timing measurements over $\sim 3$ decades have provided an upper limit[21]

$$
\begin{aligned}
\Omega_{g w}(f) & \leq 4 \times 10^{-9}\left(f / f_{T_{o b s}}\right)^{2} \\
\text { for } f & \gtrsim 1 / T_{o b s} \sim 10^{-9} \mathrm{~Hz} .
\end{aligned}
$$

These measurements are supplemented by spacecraft doppler tracking data for $f \gtrsim 10^{-6} \mathrm{~Hz}$ [22]. Spacecraft Doppler tracking has established a limit of $\Omega_{\mathrm{gw}}(f)<$ 0.025 in the band $10^{-6}-10^{-3} \mathrm{~Hz}$.

Finally, Big Bang nucleosynthesis (BBN), in combination with the observed number of neutrino families, can 
be used to infer a bound on the frequency-integrated total GW energy density [13]:

$$
\int_{f>10^{-8} \mathrm{~Hz}} \Omega_{g w}(f) d \ln f \leq 2 \times 10^{-5} .
$$

The shaded trapezoidal area in Fig. 3 indicates the magnitude a constant $\Omega_{g w}(f)$ can have, depending on the limits of integration. The dependence is weak, being $\propto \ln \left(f_{\text {upper }} / f_{\text {lower }}\right)$.

Each of these observational constraints serve to limit the range of parameters accessible to the various cosmological sources models. The source curves shown in Fig. 3 represent the maximum contribution to the stochastic $\mathrm{GW}$ background that the models can make, consistent with measurements. COBE limits the level of inflationproduced GWs; pulsar timing limits the F- and D-string model contribution at the lowest frequency range of these models; the big-bang nucleosynthesis limit constrains the pre-big bang models, since these have spectra which increase with $f$, and thus can quickly violate the BBN limit unless their upper frequency behavior is limited.

\section{Experimental technique}

The most sensitive technique for observing stochastic GWs involves cross-correlating the output of pairs of interferometric GW detectors, looking for a common source of correlated stochastic noise of non-terrestrial origin [8][9][10]. In order for the technique to be effective, the baseline between the pair of detectors must be less than the GW wavelength: $\lambda_{G W} \geq 2 D$. For the LIGO Washington-Louisiana baseline $(\sim 3000 \mathrm{~km})$, this corresponds a peak in the detection sensitivity for frequencies $f \lesssim 50 \mathrm{~Hz}$. However, the cross-correlation analysis includes all frequencies for which there is adequate signalto-noise performance (see below).

Signals from pairs of detectors are correlated using an optimal Wiener filter in the frequency domain. The cross-correlation signal is formed at discrete times, $t_{k}$, separated by the segment integration time, $T$ :

$$
Y\left[t_{k}\right]=\int \widetilde{s}_{1}^{*}(f) \widetilde{Q}(f) \widetilde{s}_{2}(f) d f ; t_{k}=k T
$$

The model-dependent optimal filter, $\widetilde{Q}(f)$, depends on the expected GW spectrum and the noise power spectra, $P_{i}(f)$, of the instruments:

$$
\widetilde{Q}(f) \propto \frac{\gamma(f) \Omega_{g w}^{\text {model }}(f)}{f^{3} P_{1}(f) P_{2}(f)} .
$$

The real function $\gamma(f)$, termed the overlap reduction factor[10], is determined by the sky-integrated average of the two-detector antenna pattern (Fig. 5). In all cases $|\gamma| \leq 1$. The sensitive frequency band of an observation is determined in part by $\gamma(f)$ and in part by the denominator of $\widetilde{Q}(f)$, containing the power spectral densities of the interferometer noise floors. Together, for the HanfordLivingston pair of interferometers, the effective band is $70 \mathrm{~Hz} \lesssim f_{L I G O} \lesssim 150 \mathrm{~Hz}$.

The implementation of the algorithm involves making many $\left(\mathrm{O}\left[10^{4}-10^{5}\right]\right)$ repeated measurements of the same quantity over shorter observation times (e.g., $T_{\text {obs }}=60 \mathrm{~s}$ ) and then averaging them over an entire run. By suitably normalizing $\widetilde{Q}(f)$, each observation generates a signal and variance estimate, $Y_{i}=\Omega_{i} T_{o b s}$ and $\sigma_{i}^{2}$. These may then be combined,

$$
\Omega_{e s t}=\frac{1}{T_{o b s}} \frac{\sum_{i} Y_{i} / \sigma_{i}^{2}}{\sum_{i} 1 / \sigma_{i}^{2}} ; \widehat{\sigma}^{-2}=\sum_{i} 1 / \sigma_{i}^{2} .
$$

The S3 search was conducted for a series of power law spectra, $\Omega_{g w}(f)=\Omega_{\alpha}(f / 100 \mathrm{~Hz})^{\alpha}$. Results are presented in Table 1. Over the course of S3, 27564 measurements, $\Omega_{i}$, were combined to determine the best estimate of $\Omega$. The experimental value, $\widehat{\Omega}_{\alpha=0}$, for S3 is consistent with zero. Thus, at the sensitivity attained during S3, $\widehat{\Omega}_{\alpha=0}$ leads to a $90 \%$ CL upper limit on $\Omega_{g w} \leq$ $8.4 \times 10^{-4}$.

The quality of the measurements may be assessed by considering the measurement residuals for the $\sim 27000$ independent measurements. These are shown in Fig. 6 and it can be seen that the residuals are consistent with unit variance normal deviates, as expected (the value of $\sigma_{\xi}=1.036$ shown in the figure includes the expected $3.6 \%$ bias correction due to the finite-duration $60 \mathrm{~s} \mathrm{seg-}$ ments used in the measurement).

\section{PROSPECTS}

As of this writing, the LIGO Scientific Collaboration is preparing to embark on a very long $(\sim 1.5$ years $)$ science run at its design sensitivity starting in the fall/winter of 2005. This S5 science run is expected to achieve the originally specified sensitivity limit to stochastic GWs for the present generation of LIGO interferometers (ref. Fig. 3).

In addition, in 2004, the United States National Science Foundation was authorized to proceed with the Advanced LIGO upgrade[23]. Design and planning for this program has begun; the expected start of the construction program is FY2008. Advanced LIGO is expected to achieve an ultimate sensitivity for a stochastic GW background of $\sim 10^{-11}$ after one year of observation.

Finally, the United States and the European Union. are collaborating on an ambitious space-based interferometer: LISA[24]. The LISA design calls for arm lengths of 


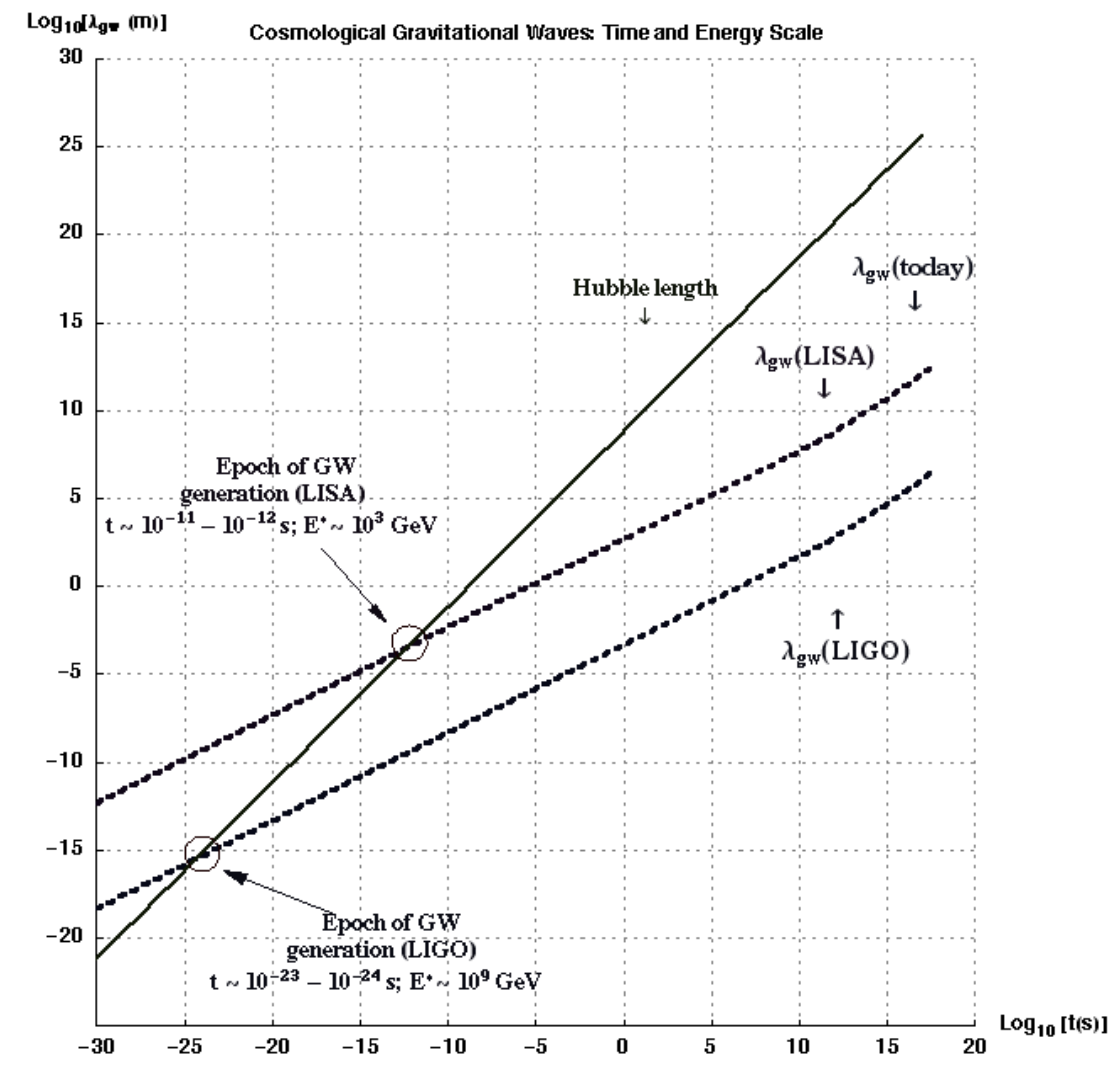

FIGURE 4. Plot of the evolution of $\lambda_{g w}$ with time.

TABLE 1. Results of the cross-correlation of LIGO's H1 and L1 interferometers, analyzed for a potential power-law stochastic background of the form: $\Omega_{\mathrm{gw}}(f)=\Omega_{\alpha}(f / 100 \mathrm{~Hz})^{\alpha}$. The frequency range for each $\alpha$ is the band that contributes $99 \%$ of the full sensitivity, as determined by the inverse variance. All results correspond to the specified band, and an observation time of $218 \mathrm{hr}$. 90\%-confidence Bayesian upper limits on $\Omega_{\mathrm{gw}}(f)$ (also expressed as limits on the strain noise density $S_{\mathrm{gw}}^{1 / 2}(f)$ ) are calculated from the point estimates and statistical errors, marginalising over a $\pm 9 \%$ and $\pm 15 \%$ uncertainty in the calibration magnitude of the H1 and L1 detectors. NOTE: upper limits on $\Omega_{g w}(f)$ include frequency-dependent factors indicated in footnote below the table. Similarly for upper limits on $S_{g w}(f)$.

\begin{tabular}{cccccccc}
\hline $\begin{array}{c}\text { Power } \\
\text { law }\end{array}$ & $\begin{array}{c}\text { Freq. } \\
\text { range }\end{array}$ & $\begin{array}{c}\text { Estimate } \\
\widehat{\Omega}_{\alpha}\end{array}$ & $\begin{array}{c}\text { Stat. Error } \\
\sigma_{\Omega_{\alpha}}\end{array}$ & \multicolumn{2}{c}{ Cal. Errors } & Upper Limit* & \multicolumn{2}{c}{ Upper Limit $^{\dagger}$} \\
\hline$\alpha=0$ & $69-156 \mathrm{~Hz}$ & $-6.0 \times 10^{-4}$ & $7.0 \times 10^{-4}$ & $\pm 11 \%$ & $\pm 15 \%$ & $8.4 \times 10^{-4}$ & $1.2 \times 10^{-23}$ \\
$\alpha=2$ & $73-244 \mathrm{~Hz}$ & $-4.7 \times 10^{-4}$ & $7.2 \times 10^{-4}$ & $\pm 11 \%$ & $\pm 15 \%$ & $9.4 \times 10^{-4}$ & $1.2 \times 10^{-23}$ \\
$\alpha=3$ & $76-329 \mathrm{~Hz}$ & $-4.0 \times 10^{-4}$ & $6.2 \times 10^{-4}$ & $\pm 11 \%$ & $\pm 15 \%$ & $8.1 \times 10^{-4}$ & $1.2 \times 10^{-23}$ \\
\hline
\end{tabular}

$* \times(f / 100 \mathrm{~Hz})^{\alpha}$

$\doteqdot \times(f / 100 \mathrm{~Hz})^{-(3-\alpha) / 2}$ 


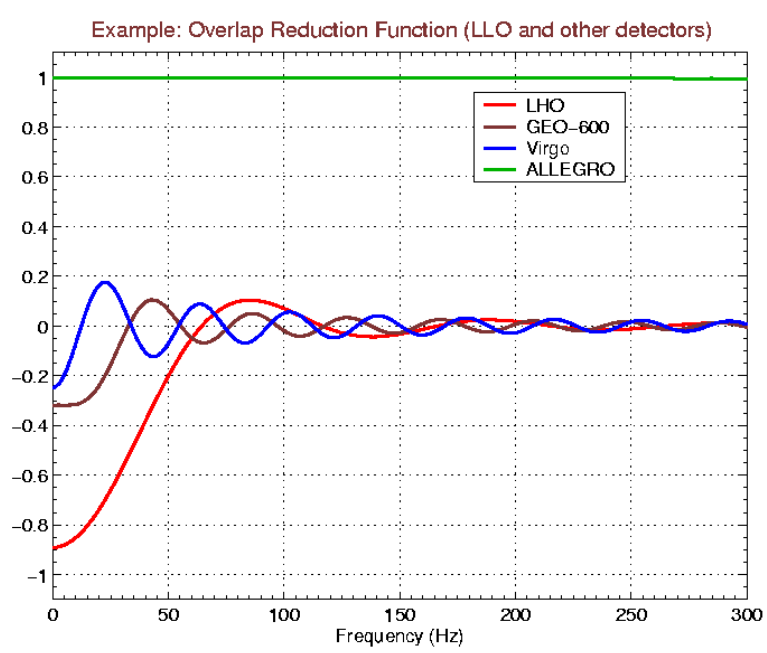

FIGURE 5. Frequency dependence of the geometrical overlap reduction factor for the LLO site and other detectors.

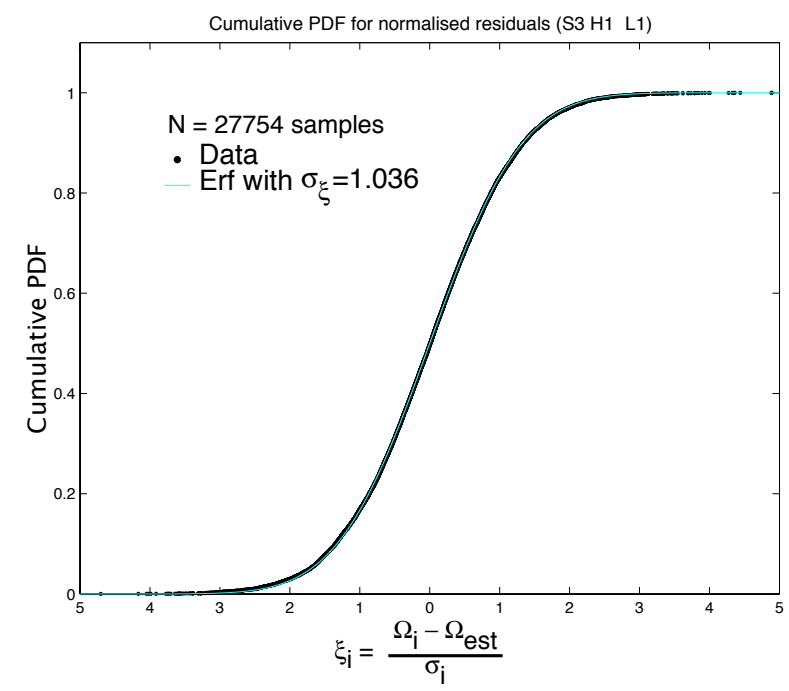

FIGURE 6. Plot of cumulative distribution of residual errors from the S3 H1-L1 analysis.

$\sim 5 \times 10^{6} \mathrm{~km}$. Consequently, the detection band spans a much lower frequency range than LIGO:

$$
10^{-3} \mathrm{~Hz} \lesssim f_{L I S A} \lesssim 10^{-1} \mathrm{~Hz}
$$

Refer to Fig. 7. The figure shows that at the lowest frequencies accessible to LISA, unresolved astrophysical foreground sources may actually limit the sensitive band. However, the $\propto 1 / f^{3}$ dependence of strain spectral density on stochastic background strength (ref. Eq. 3) implies that a space-based LISA, operating at much lower frequencies than the LIGO inteferometer sensitivity band, will be sensitive to values of

$$
2 \times 10^{-11} \lesssim \Omega_{0} \lesssim 2 \times 10^{-10}[25]
$$

\section{ACKNOWLEDGMENTS}

The author gratefully acknowledges his colleagues in the LIGO Scientific Collaboration whose work on searches for a stochastic gravitational wave background made this report possible. In addition he acknowledges the generous support of the United States National Science Foundation for the construction and operation of the LIGO Laboratory. This puiblication has been assigned LIGO Document Control Number LIGO-P050035-03. 


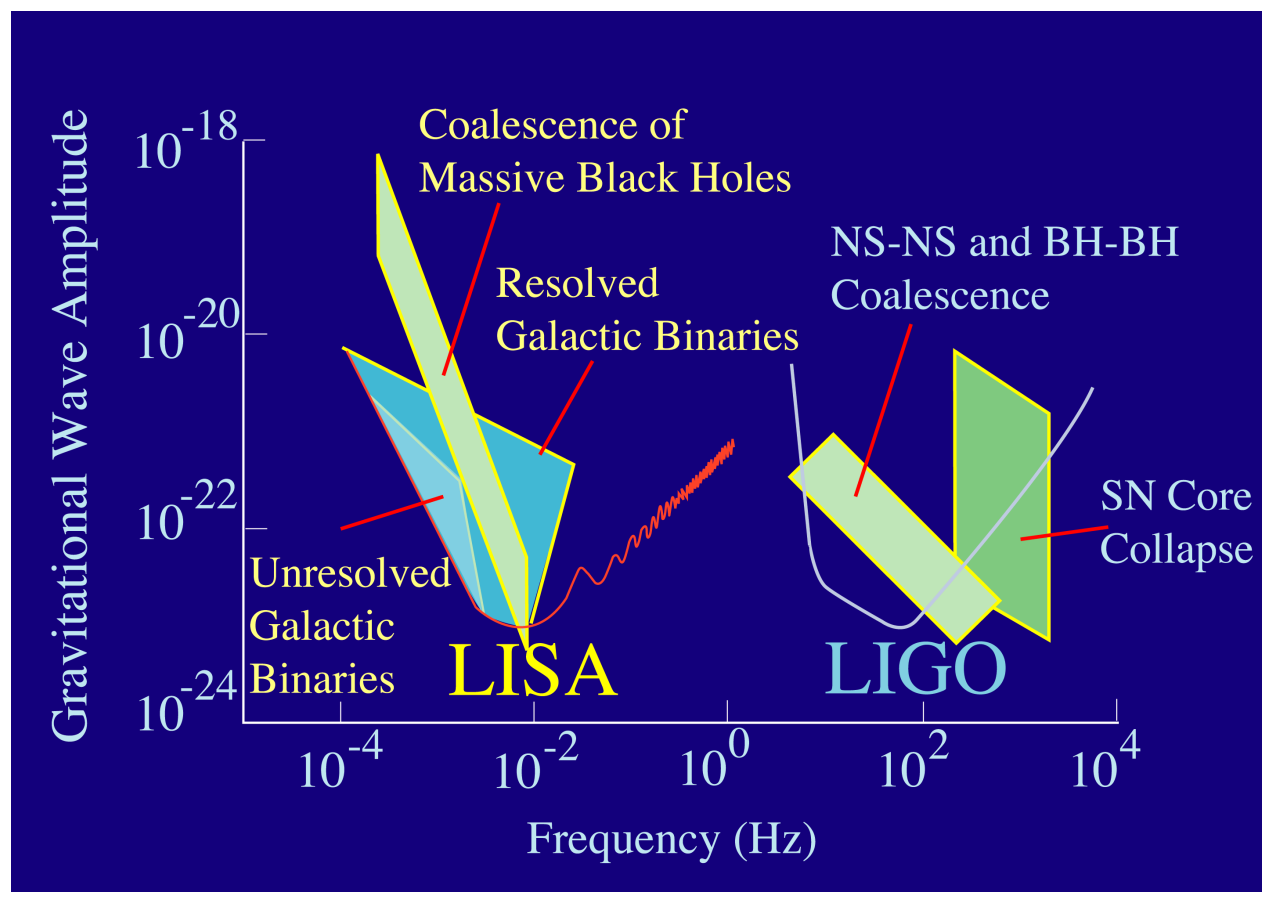

Source: NASA[26]

FIGURE 7. Schematic comparison of the frequency band and sensitivity of the space-based LISA mission and LIGO.

\section{REFERENCES}

1. Maggiore, Phys.Rept. 331 (2000) .

2. T. Regimbau, J.A. de Freitas Pacheco, http: //arxiv.org/abs/astro-ph/0105260.

3. V. Ferrari, S. Matarrese and R. Schneider, Mon. Not. R. Astron. Soc. ,303 258 (1999).

4. S. Drasco and E.E. Flannigan, Phys.Rev. D67 (2003).

5. B. Abbott et al. (The LIGO Scientific Collaboration), Phys. Rev. D 69122004 (2004).

6. B. Abbott et al. (The LIGO Scientific Collaboration), http://xxx.lanl.gov/pdf/astro-ph/ 0507254 , submitted to Phys. Rev. Lett.

7. B. Abbott et al. (The LIGO Scientific Collaboration), Nucl. Instrum. Methods A 517/1-3 154 (2004).

8. N. Christensen, Phys. Rev. D 46, 5250 (1992).

9. É.É. Flanagan, Phys. Rev. D 48, 2389 (1993).

10. B. Allen and J.D. Romano, Phys. Rev. D 59, 102001 (1999).

11. R. R. Caldwell and M. Kamionkowski, Sci. Am., 38 (January 2001).

12. A.H. Guth and S.-Y. Pi, Phys. Rev. Lett. 49, 1110 (1982).

13. Kolb and Turner, "The Early Universe", Addison-Wesley, redwood City, CA, 1990.

14. B. Allen and S. Koranda, Phys. Rev. D50 (1994).

15. Apreda et al., Nucl. Phys. B631 (2002).

16. M. Kamionkowski, Kosowski, and M. Turner, Phys. Rev. D49 (1994).

17. T. Damour and A. Vilenkin, Phys. Rev. D71 (2005)

18. M. Gasperini and G. Veneziano, Phys. Rev. D50 2519 (1994).
19. R. Brustein, M. Gasperini, M. Giovannini and G. Veneziano, Phys.Lett. B361 45 (1995).

20. A. Buonanno, M. Maggiore, and C. Ungarelli, Phys. Rev. D55 3330 (1997).

21. Lommen, astro-ph/0208572

22. Armstrong et al., ApJ 599 (2003).

23. See: http://www.ligo.caltech.edu/ advLIGO/.

24. See: http://lisa.jpl.nasa.gov/.

25. A. Vecchio, Class. Quantum Grav. 19 (2002) 1449.

26. http://lisa.jpl.nasa.gov/gallery/ images/LIGO-LISA.tif 